\title{
Segmented Motion Compensation for Complementary Coded Ultrasonic Imaging
}

\author{
Cormac Cannon, Student Member, IEEE, John Hannah, Member, IEEE, \\ and Steve McLaughlin, Senior Member, IEEE
}

\begin{abstract}
Ultrasonic imaging using complementary coded pulses offers the SNR improvements of signal coding without the filter side-lobes introduced by single-transmit codes. Tissue motion between coded pulse emissions, however, can introduce high side-lobes caused by misalignment of complementary filter outputs. This paper presents a method for filtering and motion compensation of complementary coded signals appropriate for use in medical imaging. The method is robust to the effects of non-ideal transducers on the imaging signals, includes mirrored compensation stages to reduce the impact of motion estimation error, and has been shown to reduce side-lobes to levels that compare favorably to systems using FM-coded signals of similar length and bandwidth while providing increased coding gain and range resolution. In addition, motion compensation allows the received data to be used without the frame-rate penalty usually incurred by complementary-coded imaging. The method has been verified using simulated point and speckle targets with both homogeneous and inhomogeneous motion profiles. Selected results have been verified experimentally.
\end{abstract}

\section{INTRODUCTION}

$\mathrm{S}_{\mathrm{u}}^{\mathrm{n}}$ INUSOIDAL excitation signals have traditionally been used in medical ultrasonic pulse-echo imaging systems since the advent of the technology in the 1940s. The bandwidth (and thus resolution) of such a system is inversely proportional to the duration of the transmitted pulse whereas the SNR (and dynamic range) increases in direct proportion to it, giving rise to a compromise between imaging depth and resolution. Signal coding techniques have long been discussed in the literature [1] as a method of overcoming this limitation. It is only in recent years that the ready availability of fast, inexpensive digital hardware has facilitated the development of commercial systems using coded excitation; the first of these systems were manufactured by General Electric in the late 1990s [2]. Continued growth in computing power facilitates the implementation of new techniques such as those presented in this paper.

Manuscript received July 18, 2008; accepted January 10, 2010. This work was performed as part of the BIAS consortium under a grant funded by EPSRC under their Basic Technology Programme. We acknowledge the support of the Scottish Funding Council for the Joint Research Institute with the Heriot-Watt University, which is a part of the Edinburgh Research Partnership.

The authors are with the University of Edinburgh, Institute for Digital Communications, Edinburgh, UK (e-mail: cormac@media.mit.edu).

Digital Object Identifier 10.1109/TUFFC.2010.1516

\section{A. Single-Transmit Signal Coding}

Matched filtering of individual coded signals introduces range side-lobes. In imaging applications, care must be taken with the design of coded signal and filter to keep side-lobes below about $-50 \mathrm{~dB}$ to avoid the occurrence of visible side-lobe artifacts which can offset any gain in SNR achieved by pulse compression. These issues may be exacerbated by filter mismatch caused by the effects of non-ideal transducers and of propagation through the medium. The effects of the transducer may be allowed for in the system design but those of the medium are non-linear, unpredictable, and vary with depth, presenting a less tractable problem. Published opinions vary as to the relative suitability of frequency-modulated (FM) signals and binary phase-coded (BPC) signals for medical ultrasonic imaging. A system using pseudo-chirp excitation signals, i.e., binary approximations to linear FM (LFM) chirp signals, is described in [3], however the sidelobe levels achievable with this method are of the order of $-40 \mathrm{~dB}$. An effective system using weighted LFM excitation signals and a mismatched filter is presented in [4]-[6]. Here, the effects of frequency-dependent attenuation are modeled as a downward shift in the center frequency of the pulse and it is concluded that the LFM signal is more robust to this effect than either non-linear FM (NLFM) or BPC signals on the basis of its ridge-shaped ambiguity function. A method for the design of NLFM signals matched to the transducer bandwidth using least squares optimization is presented in [7], with results indicating that the effects of non-linear attenuation are no more severe for NLFM than for LFM, whereas excellent side-lobe performance is achievable without the weighting of filter coefficients required for LFM. This latter consideration, combined with the matching of the transducer bandwidth, results in an increase in SNR, though the bandwidthmatching results in a slight reduction in axial resolution at low depths. Both LFM- and NLFM-based approaches, however, require the use of multi-level pulsers and exhibit some broadening and lowering of the main-lobe response with increasing depth. High time-bandwidth (TB) products of the order of 50 or more are required to reduce FM side-lobes to levels suitable for imaging, and these long signals can introduce problems with dynamic focusing and time-gain-control [8].

In systems using BPC signals, an oversampled representation of a binary code is generally used to phase modulate a base sequence or chip. An advantage of this approach is that it may be implemented using inexpensive 
bi-polar pulsers. The received pulses may be processed using a decoding filter matched only to the binary code, thus lumping the effects of the transducer response and frequency-dependent attenuation in with the base sequence [9]. A system using BPC signals and extended spiking filters derived by spectrum inversion is reported in [10], and spiking filters for Barker-coded excitation are described in [11]. The method is effective, although the maximum coding gain achievable is limited to $11 \mathrm{~dB}$ by the maximum Barker code length of 13 and relatively long filters are required to bring side-lobe levels below the -50 to $-80 \mathrm{~dB}$ typically required for medical imaging.

\section{B. Complementary Coded Ultrasound}

Complementary sets of binary codes, like the wellknown Golay pairs [12], offer the benefits of binary-phasecoding without the filter side-lobes introduced by singletransmit codes. Members of a complementary code set may have auto-correlation side-lobes higher than those of some other classes of binary code. When summed, however, they cancel completely. In complementary code-based imaging, the individual coded signals of a set are usually transmitted separately during discrete acquisition cycles. Any motion of the subject between acquisition cycles will introduce residual side-lobes caused by misalignment of filter outputs and will broaden and lower the main lobe of the combined filter response.

The conventional approach to ultrasonic Golay imaging is to transmit both codes sequentially in the same direction before acquiring data for the next transmission focus, thus halving the system frame rate. This assumes the inter-pulse interval is small enough to cause only minor misalignment. In [9], [13], [14], two orthogonal Golay code pairs and simultaneous transmission were used to avoid a reduction in frame-rate, but the motion issue remains. Other work [15] suggests exploitation of a property of the 2-bit Golay code pair to suppress motion artifacts. Received reflections corresponding to each pulse of the Golay-coded pair are filtered using both codes of the complementary pair. The additional filter outputs are subtracted from the combined Golay filter envelope and apparently cancel misalignment side-lobes. This approach appears to involve gating of decoded signals, meaning cancellation will only be partial for the overlapping reflections encountered in most imaging scenarios. In addition, this cancellation only occurs for the 2-bit Golay pair and fails to compensate for the reduction in compression and axial resolution caused by spreading and lowering of the main-lobe. A detailed comparison of LFM and Golay-coded excitation signals for use in ultrasonic imaging may be found in [9]. The effects of frequency-dependent attenuation and non-linearity on sidelobe levels and SNR are considered, as are those of tissue motion. Results are comparable for the two waveforms. The Golay-coded pair provides a higher coding gain than an LFM signal of similar length as the effective length of a complementary code set is the sum of the lengths of its constituent codes [16], although motion can introduce severe side-lobe artifacts.

This paper describes a novel approach to complementary-coded ultrasonic imaging, whereby the constituent codes of a complementary set are transmitted individually without reducing frame rate. Section II gives an overview of the system and a high-level description of the signal flow. Section III describes the simulation environment and experimental facilities used to evaluate it. Baseline results comparing the performance of complementary-coded imaging in the absence of motion to that of other signal coding schemes current in the literature are presented in Section IV. Section V describes the effects of motion of the subject between emissions and introduces a method of motion-compensation. This method is extended in Section VI to allow compensation of inhomogeneous motion, i.e., situations in which a single B-mode image line contains scattering centers with different velocities. Section VII presents some conclusions and plans for further research.

\section{System OutLINe}

Consider a B-mode image consisting of $N_{\mathrm{l}}$ image lines and using $N_{\mathrm{f}}$ transmit focal depths per line. Using an uncoded pulse or single-transmit code, $N_{\mathrm{f}}$ acquisitions are required to form each image line and $N_{\mathrm{f}} N_{\mathrm{l}}$ acquisitions to form the full image. Using a set of $N_{\mathrm{c}}$ complementary codes, the number of acquisitions required to form a single image becomes $N_{\mathrm{c}} N_{\mathrm{f}} N_{\mathrm{l}}$. Conventionally, every code of the set is transmitted in each focal direction in turn, i.e., $N_{\mathrm{c}}$ time-adjacent acquisitions will be required for a single focal depth and image line, while $N_{\mathrm{c}} N_{\mathrm{f}}$ time-adjacent acquisitions are used to form each full image line. This ensures that the complementary ultrasonic reflections that are combined to form the B-mode image for a given focal depth and image line are acquired $T_{\mathrm{PRF}}$ seconds apart, where $T_{\mathrm{PRF}}$ is the pulse repetition period, thus minimizing the effects of motion on complementary side-lobe cancellation.

Using the proposed method, data for an entire image is acquired using each code of the set in turn. As complementary acquisitions for each transmission focus occur $N_{\mathrm{f}} N_{\mathrm{l}} T_{\mathrm{PRF}}$ seconds apart, tissue motion can introduce significant visual artifacts caused by partial side-lobe cancellation. This may, however, be counteracted by re-aligning the received data using estimates of the motion so that the side-lobes cancel appropriately. Using this approach, $N_{\mathrm{c}} N_{\mathrm{f}} N_{\mathrm{l}}$ acquisitions are required to form the first B-mode image but a new image is formed after each subsequent $N_{\mathrm{f}} N_{\mathrm{l}}$ acquisitions as the acquired data may be used recursively, allowing complementary coded imaging to be employed without incurring a frame-rate penalty. A similar method was proposed by Nikolov [17] for high frame-rate synthetic aperture imaging using single-transmit linearFM coded excitation signals.

In the interests of clarity, all block diagrams and mathematical descriptions refer to the signal flow for a single 
line (or transmission direction) of a B-mode image using a fixed receive focus. Scaling for multiple lines is a straightforward extension.

\section{A. Transmit Coding}

Let $C(n, l)$ represent the bi-phase representation of a complementary set of $N$ binary codes, each of length $L$, and $b(t)$ represent a chip or basis sequence of duration $T_{\mathrm{b}}$.

$$
C(n, l) \in(-1,1) \ldots, \quad 1<n<N, \quad 1<l<L
$$

The set of excitation signals, $E_{N}(n, t)$, may be written

$$
E_{N}(n, t)=c_{N}(n, t) * b(t), \quad 1 \leq n \leq N,
$$

where

$$
c_{N}(n, t)=\sum_{l=1}^{L} C(n, l) \delta\left(t-l T_{\mathrm{b}}\right), \quad 1 \leq n \leq N .
$$

The individual signals of the set $E_{N}(n, t)$ are transmitted in a repeating series such that the signal transmitted at the beginning of the $i$ th acquisition cycle $E(i, t)=E(i-$ $N, t)$, and

$$
E(i, t)=E_{N}(\bmod (i-1, N)+1, t)
$$

Defining $c(i, t)$ in a similar fashion,

$$
c(i, t)=c_{N}(\bmod (i-1, N)+1, t) .
$$

In the current system, the two codes of a Golay pair are used to modulate a basis sequence consisting of a singlecycle square wave at the transducer center frequency, $F_{\mathrm{c}}$, allowing the final shape of the transmitted waveform to be dictated by the transducer frequency response. The resulting signals are transmitted during alternate acquisition cycles.

\section{B. Receive Processing}

The receive signal flow is illustrated in Fig. 1. Using a fixed receive focus, the beam-formed reflection from the ith transmitted pulse is given by

$$
\begin{aligned}
S(i, t) & =E(i, t) * A_{\mathrm{T}}(t) * R(i, f, t) * A_{\mathrm{R}}(t)+N_{\mathrm{R}}(i, t) \\
& =E(i, t) * A_{\mathrm{TR}}(t) * R(i, f, t)+N_{\mathrm{R}}(i, t),
\end{aligned}
$$

where $A_{\mathrm{T}}(t)$ and $A_{\mathrm{R}}(t)$ represent the impulse responses of the transmit and receive apertures, repectively, $A_{\mathrm{TR}}(t)$ is their combined response, $N_{\mathrm{R}}(i, t)$ is a lumped noise term, and $R(i, t)$ represents the reflectivity function of the region insonated by the beam during the $i$ th acquisition expressed as a function of time. Combining (4) and (6),

$$
\begin{aligned}
S(i, t) & =c(i, t) * b(t) * A_{\mathrm{TR}}(t) * R(i, t)+N_{\mathrm{R}}(i, t) \\
& =c(i, t) * B(t) * R(i, t)+N_{\mathrm{R}}(i, t),
\end{aligned}
$$

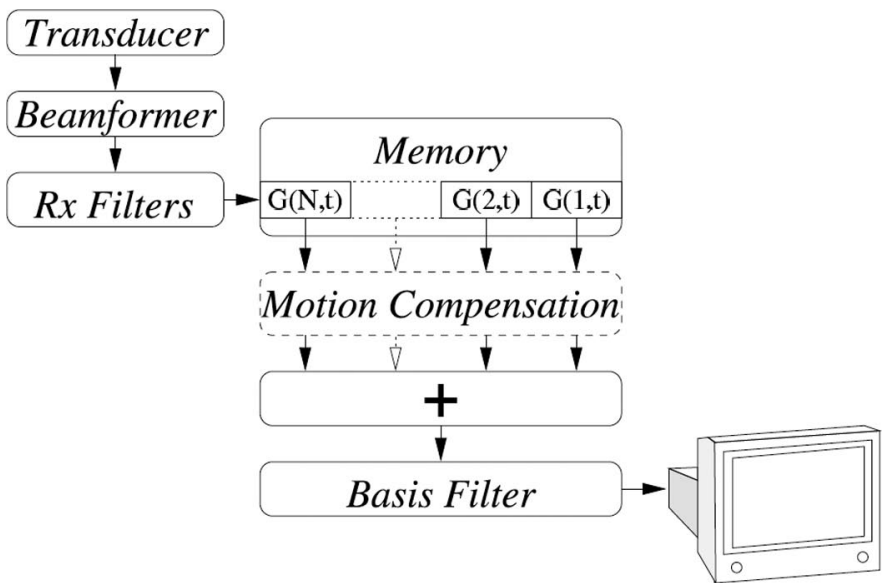

Fig. 1. Receive signal flow for a motion-compensated complementarycoded imaging system.

where $B(t)$ represents the convolution of the basis sequence with the combined transmit and receive aperture response.

When using dynamic receive focusing and apodisation, $A_{\mathrm{R}}(t)$ varies as a function of imaging depth and the convolution in (7) is no longer time-invariant. In these circumstances, filtering should ideally take place before beam-formation, a process known as pre-compression. In practical implementations, hardware costs can be reduced significantly using post-compression, i.e., reversing the order of these operations. This introduces an error that depends on a range of factors including focal depth, signal length, the distance between focal points [8] and the f-number used [18].

Filtering of the received ultrasonic reflections is split into two stages. In the first, received signals are passed through a compression filter whose coefficients are an oversampled version of the appropriate binary code [9],

$$
\begin{gathered}
F(i, t)=c\left(i, N T_{\mathrm{b}}-t\right) \\
=F(\bmod (i-1, N)+1, t), \\
F(i, t) * c(i, t)=c_{i i}(i, t),
\end{gathered}
$$

where $c_{i i}(i, t)$ denotes the auto-correlation sequence of $c(i, t)$.

No matching of the basis sequence is attempted at this stage, thus insulating the binary code filters from the distorting effect of the medium and transducer and preserving the complementary nature of the filter outputs. The output of this first filter stage is

$$
\begin{aligned}
G(i, t) & =F(i, t) * S(i, t) \\
& =c_{i i}(i, t) * B(t) * R(i, t)+N_{\mathrm{G}}(i, t),
\end{aligned}
$$

where $N_{\mathrm{G}}(i, t)=F(i, t) * N_{\mathrm{R}}(i, t)$.

The sum of $N$ adjacent complementary filter outputs is 


$$
\begin{aligned}
C_{\mathrm{U}}(i, t) & =\sum_{a=i-N+1}^{i} G(a, t) \\
& =\sum_{a=i-N+1}^{i} c_{a a}(a, t) * B(t) * R(a, t)+N_{\mathrm{C}}(i, t) \\
& =B(t) * \sum_{a=i-N+1}^{i} c_{a a}(a, t) * R(a, t)+N_{\mathrm{C}}(i, t),
\end{aligned}
$$

where $N_{\mathrm{C}}(i, t)=\sum_{a=i-N+1}^{i} N_{\mathrm{G}}(a, t)$.

If the region within the beam remains static between acquisition cycles, $R(i, t)$ becomes $R(t)$ and (11) may be simplified to

$$
\begin{aligned}
C_{\mathrm{U}}(i, t) & =B(t)^{*} R(t) * \sum_{a=i-N+1}^{i} c_{a a}(a, t)+N_{\mathrm{C}}(i, t) \\
& =N \delta\left(t-N T_{\mathrm{b}}\right) * B(t) * R(t)+N_{\mathrm{C}}(i, t) .
\end{aligned}
$$

Because the components of $N_{\mathrm{G}}$ are uncorrelated, $N_{\mathrm{C}}(i, t)$ and $N_{\mathrm{G}}(i, t)$ are of equal power and, in the absence of motion, the coding gain is $10 \log _{10}(N) \mathrm{dB}$.

Let $M(i, n, t), 1 \leq n \leq N$, represent the outputs of the motion compensation block at the ith emission, with $M(i, N, t)=G(i, t)$. The role of the motion compensation block is to ensure that the sum of compensated outputs $C(i, t)$ approaches

$$
\begin{aligned}
C(i, t) & =\sum_{n=1}^{N} M(i, n, t) \\
& \cong B(t) * R(i, t) * \sum_{a=i-N+1}^{i} c_{a a}(a, t)+N_{\mathrm{C}}(i, t) \\
& =N \delta\left(t-N T_{\mathrm{b}}\right) * B(t) * R(i, t)+N_{\mathrm{C}}(i, t) .
\end{aligned}
$$

Some ways in which this may be accomplished are described in Section V.

The sum of complementary-code filter outputs is passed through a final filter matched to $b_{\mathrm{TR}}$. Separation of the receive filtering into two stages reduces its computational cost significantly. The long compression filters consist of just $L \pm 1$-valued coefficients separated by zeros and may be implemented using $(L-1)$ addition/subtraction operations at each time-step, whereas the basis filter is of the order of $1 / L$ times the length of the coded excitation sequences. The compression filters are protected from mismatch effects caused by propagation through the medium and transducer, which could potentially be dealt with using a depth-dependent basis filter.

\section{Evaluation Methodology}

The B-mode imaging system described in Section II has been evaluated by simulation using Matlab (The MathWorks, Natick, MA) and the Field II ultrasound simula- tion tool [19]. Some simulation results have been verified experimentally in collaboration with our research partners in the BIAS (Biologically Inspired Acoustic Systems) consortium. Both simulations and experiments compared the performance of the proposed motion-compensated, complementary-coded pulses with those of linear-FM pulses and an uncoded wide-band sinusoidal pulse. The measure of axial resolution employed throughout is the $-6 \mathrm{~dB}$ main-lobe width expressed in terms of $\lambda=c / B_{\text {tr }}$, where $c$ is the average speed of sound in tissue is and $B_{\text {tr }}$ is the $-6 \mathrm{~dB}$ bandwidth of the transducer.

\section{A. Simulation Environment}

The simulation results presented here all used a 192-element linear array transducer with a $7.5 \mathrm{MHz}$ center frequency and a fractional bandwidth of $83.3 \%$. The sampling frequency for the simulated ultrasound system was set to $150 \mathrm{MHz}$. Those simulations including the effects of attenuation used frequency-dependent attenuation of $0.5 \mathrm{~dB} / \mathrm{MHz} / \mathrm{cm}$. All of the simulations used a Hanning apodisation weighting, dynamic receive focusing, and an f-number of 2 on transmit and receive. Three transmit focal depths were used for the B-mode imaging and motioncompensation simulations, and ten were used for the filter envelope simulations in Section IV to minimize the impact of variation in focusing settings on the results. The simulated system currently uses post-compression, although motion compensation is equally applicable to a system using pre-compression.

Two types of phantom were used in simulation. The first consisted of 10 simulated point scatterers positioned at 10-mm intervals between 5 and $95 \mathrm{~mm}$ from the transducer along its central axis and was used to compare filter envelope characteristics under a range of conditions. The second was a simulated cyst phantom and was used to compare the performance of the signal coding schemes on a more realistic imaging target. The cyst phantom was based on one of the code examples provided with Field II. It contained 7 high-intensity point targets, 7 high-intensity scattering regions of increasing diameter and 7 empty regions of decreasing diameter in a volume measuring 35 $\times 5 \times 80 \mathrm{~mm}$ and containing 50000 scatterers.

\section{B. Experimental Verification}

Some preliminary experiments were carried out using an experimental configuration under development by members of the Medical Physics research group at the Division of Medical and Radiological Sciences, University of Edinburgh. These experiments were designed to verify the robustness of motion compensated complementary-coding to the effects of transmission using non-ideal transducers and used a single unfocused transducer with a center frequency of $5 \mathrm{MHz}$ suspended in a water tank. The transducer mounting could be actuated electronically in two dimensions and reflected signals recorded using a data acquisition board and Labview (National Instruments, 
Austin, TX). Some results obtained using this facility are presented in Section V, although ongoing development of the system made it impossible to obtain a complete set of results.

\section{Signals and Filters}

The LFM chirp and weighted matched filter were generated as described in [5]. The modified Elliptical-Tukey window of (14) was applied to the LFM signal to reduce distant side-lobes associated with Fresnel ripples in the signal spectrum.

$$
W_{\mathrm{et}}(n)= \begin{cases}\frac{\sqrt{1-x^{2}}}{2}\left[1+\cos \left(4 \pi\left(\frac{n N}{N-1}-\frac{1}{4}\right)\right)\right] & 0 \leq n \leq a \\ \sqrt{1-x^{2}} & a<n<b \\ \frac{\sqrt{1-x^{2}}}{2}\left[1+\cos \left(4 \pi\left(\frac{n N}{N-1}-\frac{3}{4}\right)\right)\right] & b \leq n<N,\end{cases}
$$

where

$$
\begin{gathered}
a=\text { floor }(R(N-1))+1 \\
b=N-a+1 \\
x=-1+\frac{2 n}{N-1} .
\end{gathered}
$$

This was originally proposed in [20] and reduces side-lobe levels further than the Tukey window proposed in [5] at the expense of a $<10 \%$ increase in main-lobe width. A window factor [denoted by $R$ in (15)] of 0.25 was used. The mismatched filter coefficients were obtained by timereversal of the LFM pulse convolved with the transducer impulse response and weighted with the Dolph-Chebyshev window [21], [22] given by the equation

$$
W_{\mathrm{ch}}(n)=\frac{1}{N}\left[1+2 S \sum_{m=1}^{M} T_{2 M}\left(x_{0} \cos \frac{\theta_{m}}{2}\right) \cos m \theta_{n}\right],
$$

where $N=2 M+1,-M \leq n \leq M ; \theta_{a}=(2 a \pi) / N$; and $S$ is a side-lobe suppression term [a value of $20 \log _{10}(-90)$ was used during this work]. $T_{2 M}(\ldots)$ is the $2 M$ th term of the Chebyshev polynomial defined by the equations

$$
T_{a}(x)= \begin{cases}\cos \left(a \cos ^{-1} x\right) & |x| \leq 1 \\ \cosh \left(a \cosh ^{-1} x\right) & |x|>1\end{cases}
$$

Note that (18) is valid only for odd values of $N$.

The LFM pulse and filter used in simulations may be seen in Figs. 2(a) and 2(b). The signal sweeps a $9.4 \mathrm{MHz}$ bandwidth centered at $7.5 \mathrm{MHz}$. A pulse duration of $10.7 \mu \mathrm{s}$ was chosen to match that of an 80-bit binaryphase coded signal. The resulting signal has a time-bandwidth product of 100, ensuring excellent side-lobe performance. Figs. 2(c) and 2(d) show the central portions of the amplitude envelopes of the matched and mismatched filter responses to the signal, both with and without the

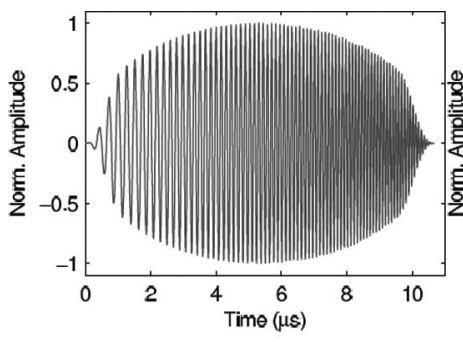

(a) Linear FM Signal

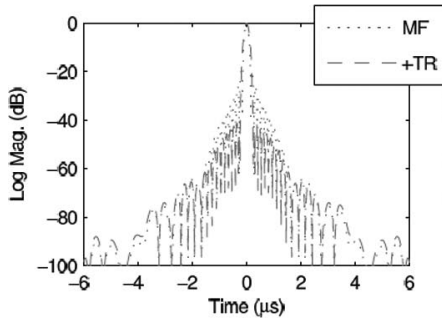

(c) Matched filter response

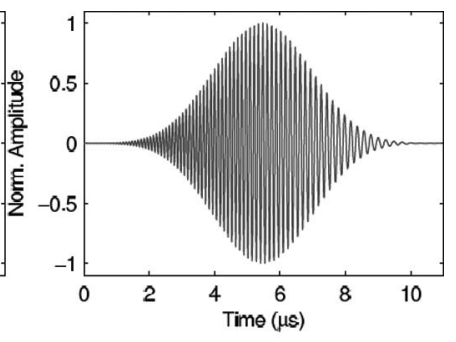

(b) Mismatched Filter

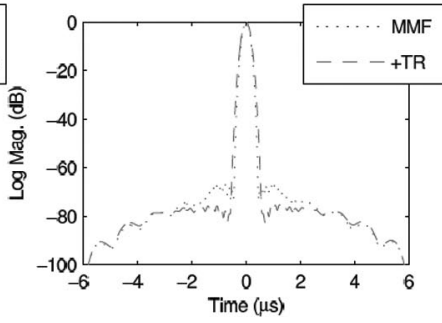

(d) Mismatched filter response
Fig. 2. Linear FM signal, mismatched filter, and normalized amplitude envelopes of the matched filter (MF) and mismatched filter (MMF) responses with and without transducer response (TR).

table I. Golay Code Pairs Used in Simulation.

\begin{tabular}{ll}
\hline Length & Code pair \\
\hline 40 & 6B 9AE7 E606 \\
& 94 5AE8 1A06 \\
80 & 6B9A E6BA 517E 5F98 19F9 \\
& 6BA5 16B9 AE7E 6068 1A06 \\
\hline
\end{tabular}

effects of the bi-directional transducer impulse response. Neglecting transducer effects, the matched filter envelope has a $-6 \mathrm{~dB}$ resolution of $1.1 \lambda$ and peak side-lobes at $-20 \mathrm{~dB}$ compared with values of $1.9 \lambda$ and $-67 \mathrm{~dB}$ for the mismatched filter. Convolution with the bidirectional transducer response reduces the peak matched filter sidelobes to $-41 \mathrm{~dB}$ and those of the mismatched filter to $-76 \mathrm{~dB}$ while increasing their main-lobe widths to $1.4 \lambda$ and $2.2 \lambda$, respectively.

The complementary-coded signal pairs used in the simulations were generated by using the 40- and 80-bit Golay code pairs of Table I to phase-modulate a singlecycle square-wave chip at the transducer center frequency of $7.5 \mathrm{MHz}$, producing coded signals of duration $5.3 \mu \mathrm{s}$ and $10.7 \mu \mathrm{s}$. The 80-bit pulse is shown in Fig. 3(a). The resulting signals were then low-pass filtered to reduce the likelihood of aliasing issues affecting simulation results. In a real system, this might reduce heating effects associated with dissipation of high-frequency energy outside the transducer bandwidth. The chip is shown in Fig. 3(b), both with and without the effects of the low-pass filter and the transducer. The same square-wave chip was also used as the uncoded pulse in simulations. The 80-bit Golay pulse pair was used for the simulations reported in Section IV to prevent differences in signal length from affecting the comparison of FM and Golay performance in the presence of attenuation. The 40-bit pulse pair was 


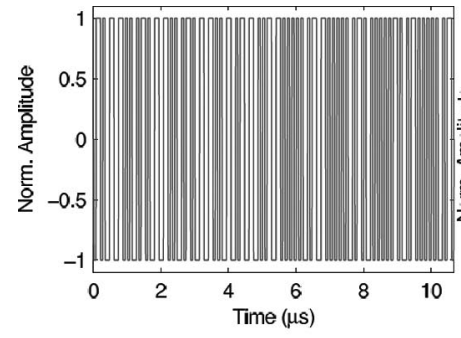

(a) 80-bit Golay coded pulse

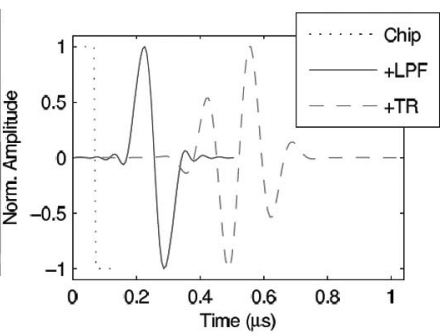

(b) Chip

Fig. 3. Golay-coded pulse and square-wave chip. The chip is shown with and without the effects of low-pass filtering (LPF) and the transducer (TR).

used for the simulations reported in Sections V and VI, as longer signals can reduce the efficacy of motion compensation in the presence of the inhomogeneous motion described in Section VI and a complementary-coded signal pair provides the coding gain of single-transmit signal of the same bandwidth and twice the length. The simulated B-mode images were all generated using the 40-bit pulse pair.

The complementary signals used in experiments were generated using Golay code pairs of lengths 40 and 16 to modulate a square-wave chip at the transducer center frequency of $5 \mathrm{MHz}$, yielding signal lengths of $8 \mu \mathrm{s}$ and $3.2 \mu \mathrm{s}$.

The magnitude spectra of the LFM, uncoded, and Golay-coded pulses and those of the sum of compressionfiltered Golay pulses may be seen in Fig. 4. These have been shown with and without the effects of low-pass filtering and the transducer. The spectrum of the LFM signal is band-limited, whereas those of the remaining signals contain significant amounts of energy at high-frequencies. Low-pass filtering and convolution with the bi-directional transducer response reduce this significantly, and the spectrum of the received signals is largely dictated by the frequency response of the transducer. Comparison of Figs. 4(b) and 4(d) shows that the spectrum of the sum of compression-filtered Golay pulses is almost identical to that of the chip.

The basis filter employed for the complementary-coded and uncoded excitation pulses was obtained by convolving a time-reversed chip with a bandpass filter matched to the transducer.

\section{Coded Imaging Performance Without Motion}

In the absence of attenuation, neither Golay or LFM envelope characteristics are adversely affected by increasing depth, although variations in focusing geometry and apodisation settings result in a slight depth-dependent variation in peak signal levels for all three types of excitation pulse. The combined envelope of the Golay-coded signal pair exhibits no side-lobes, and both it and the uncoded single-cycle pulse have a mean $-6 \mathrm{~dB}$ main-lobe width of $1.4 \lambda$. The LFM filter output exhibits side-lobes at $-75 \mathrm{~dB}$ and has a $-6 \mathrm{~dB}$ main-lobe width of $2.2 \lambda$.

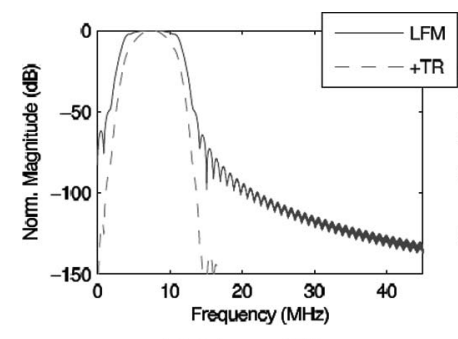

(a) Linear FM

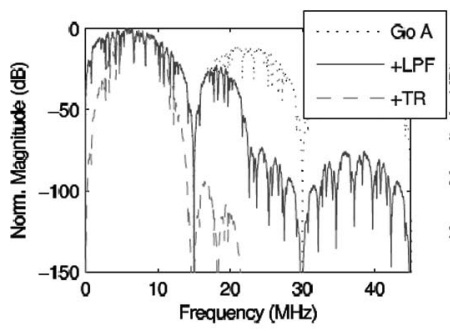

(c) Un-compressed Golay pulse

Fig. 4. Magnitude spectra of signas used in sintation, both with and without the effects of low-pass filtering (LPF) and the transducer (TR).

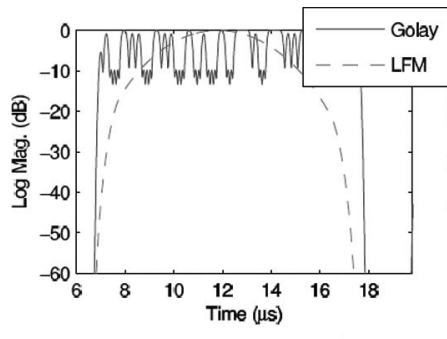

(a) Signal envelopes - $5 \mathrm{~mm}$

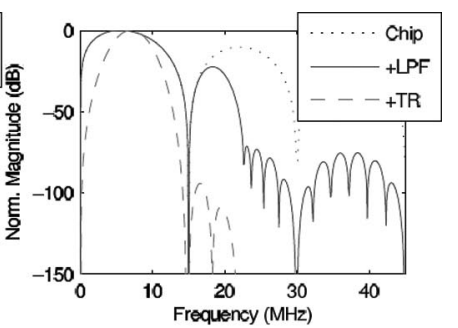

(b) Chip / uncoded pulse

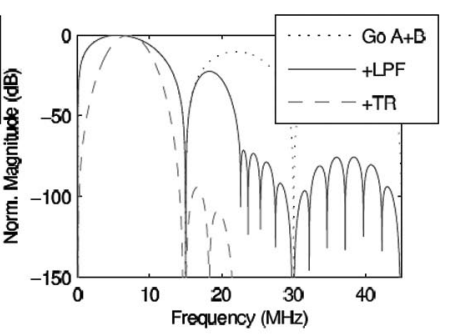

(d) Sum of compressed Golay pulses

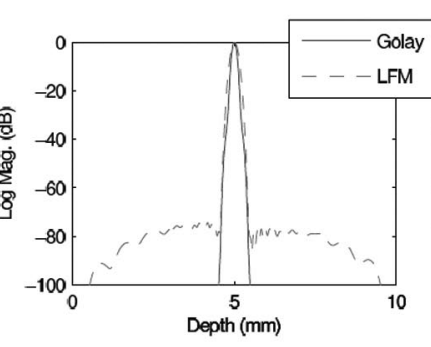

(c) Filtered envelopes - $5 \mathrm{~mm}$

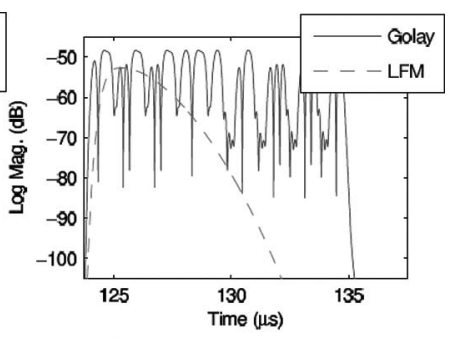

(b) Signal envelopes - $95 \mathrm{~mm}$
Fig. 5. Effects of simulated attenuation on linear FM (LFM) and Golay pulses reflected from $5 \mathrm{~mm}$ and $95 \mathrm{~mm}$ and corresponding filter response envelopes.

The introduction of simulated frequency-dependent attenuation of $0.5 \mathrm{~dB} / \mathrm{MHz} / \mathrm{cm}$ results in a depth-dependent reduction in received signal level, as shown in Figs. 5(a) and $5(\mathrm{~b})$. This is slightly more severe for the LFM signal than for the Golay coded and uncoded pulses, as the lower frequency components least affected by attenuation have already been reduced by the amplitude weighting necessary for FM side-lobe reduction. As a result, the peak level of the reflected LFM envelope from $95 \mathrm{~mm}$ is $4 \mathrm{~dB}$ below that of the Golay-coded and uncoded pulses.

The effects of attenuation are negligible at a depth of $5 \mathrm{~mm}$, as may be seen in Fig. 5(c). The LFM signal exhibits side-lobes at $-75 \mathrm{~dB}$. The $-6 \mathrm{~dB}$ widths of the main- 


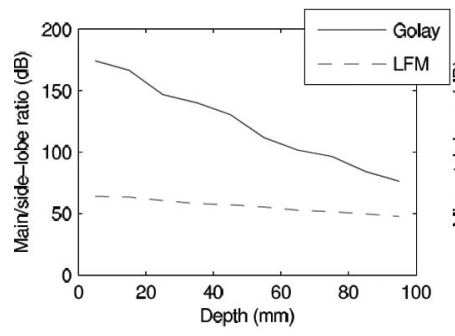

(a) Main-lobe/side-lobe ratio

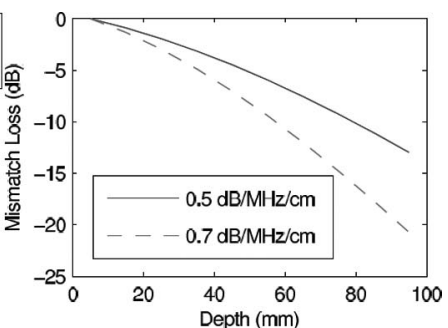

(b) Mismatch loss for linear FM pulses
Fig. 6. Effects of attenuation and imaging depth on the filter envelope characteristics of a Golay coded signal pair and linear FM (LFM) chirp.

lobes are unchanged. At a depth of $95 \mathrm{~mm}$, the effects of attenuation are evident. The LFM side-lobes have risen to $-51 \mathrm{~dB}$, whereas the combined Golay filter envelope exhibits side-lobes at $-91 \mathrm{~dB}$ (note that these appear to be caused by aliasing in the Field II simulator's implementation of frequency-dependent attenuation). The main-lobe width of the Golay envelope has increased by $29 \%$ to $1.8 \lambda$, as has that of the uncoded pulse envelope, whereas that of the LFM envelope has increased by $5 \%$ to $2.3 \lambda$. The depth-dependent effects of attenuation on side-lobe levels are plotted in Fig. 6(a).

The peak of the LFM filter envelope at $95 \mathrm{~mm}$ is $65 \mathrm{~dB}$ lower than that at $5 \mathrm{~mm}$. The corresponding difference for the uncoded pulse and sum of compression-filtered Golay-coded pulses is $52 \mathrm{~dB}$. This $13 \mathrm{~dB}$ mismatch loss is due to the amplitude weighting applied to the transmitted LFM pulse and filter coefficients, both of which emphasize the frequency components at the center of the pulse (in both time and frequency, because of the time/frequency domain symmetry of LFM pulses). At depth, these components are heavily attenuated as illustrated in Fig. 5(b) and compression is considerably less effective. Using an unweighted linear FM chirp and matched filter under identical conditions, the mismatch loss is of the order of $2 \mathrm{~dB}$ because equal weight is given to the entire range of frequencies. Binary-phase coded pulses are unaffected by this phenomenon because the two constituent coded symbols have identical magnitude spectra. Compression is a matter of simply shifting and adding equally spaced samples, inverting the sign as appropriate.

The overall peak reduction caused by attenuation, focusing variation, and filter mismatch was estimated by comparing the peak envelope values of filtered reflections from scatterers at $15 \mathrm{~mm}$ up to $95 \mathrm{~mm}$ to that at $5 \mathrm{~mm}$ for each pulse type. The results obtained using the uncoded pulse were used as an estimate of the loss caused by attenuation and focusing variation and subtracted from the estimates for the coded pulse types to obtain an estimate of mismatch loss. Estimated LFM mismatch loss has been plotted against depth in Fig. 6(b) for simulated attenuation of $0.5 \mathrm{~dB} / \mathrm{MHz} / \mathrm{cm}$ and $0.7 \mathrm{~dB} / \mathrm{MHz} / \mathrm{cm}$. The Golay results showed no evidence of mismatch loss.

The B-mode images in Fig. 7 illustrate the effects of mismatch loss on high-frequency imaging. At depths of
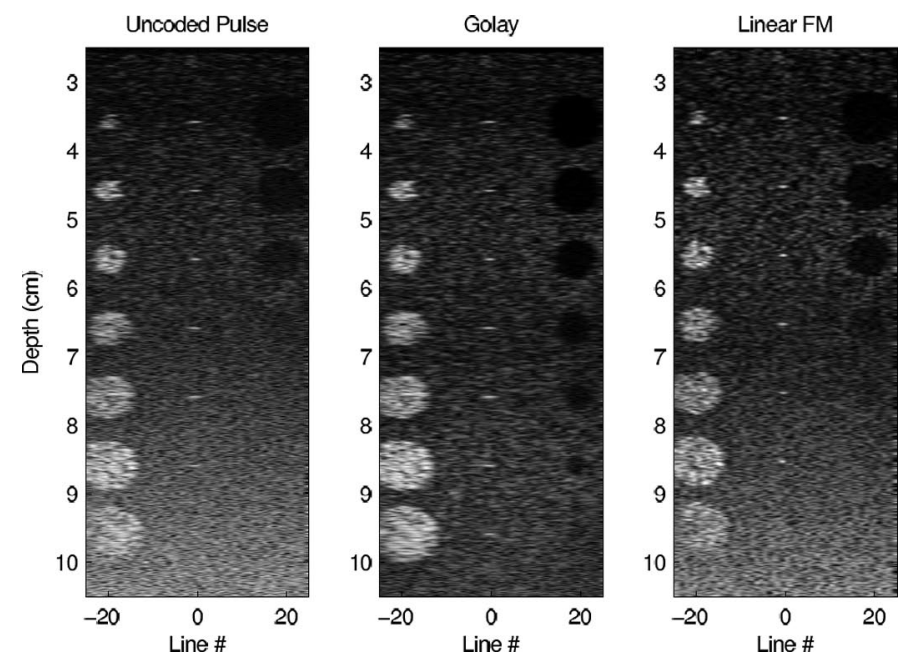

Fig. 7. Simulated B-mode images of the cyst phantom acquired using an uncoded pulse, a pair of 5.3- $\mu$ s Golay-encoded pulses, and a 10.7- $\mu \mathrm{s}$ linear FM (LFM) pulse. $45 \mathrm{~dB}$ additive noise.

60 to $70 \mathrm{~mm}$, the images obtained using Golay and LFM pulses are both clearer than that obtained using the uncoded pulse. At depths beyond $85 \mathrm{~mm}$, the SNR benefits of compression are still evident in the Golay image, whereas mismatch loss has exceeded the coding gain provided by the LFM pulse and it no longer offers a discernible advantage over the uncoded wide-band pulse.

\section{Homogeneous Motion and Compensation}

Ultrasonic back-scatter from blood is relatively low in amplitude and blood flow estimation usually requires rapid transmission of several pulses in the same focal direction and averaging of multiple estimates to reduce error. The relatively long period between complementary emissions would render blood-flow estimation impractical but tissue motion velocities tend to be significantly lower than those typical of blood flow and back-scatter from tissue is much higher in magnitude.

A change in position of a scatterer element between complementary sequence transmissions will induce a relative change in time of arrival of subsequent reflections from that element, causing misalignment between the filtered reflections corresponding to individual signals of the set. Assuming the motion of all scatterers in the region insonated by the beam is homogeneous and in the direction of the transducer axis, define $\tau_{a}^{b}$ as the round-trip delay corresponding to the change in position of the scatterers between the $a$ th and $b$ th acquisitions from a single focal region. It is then possible to relate the reflectivity functions of the region insonated by the beam during the two acquisition intervals:

$$
R(b, t) \cong R\left(a, t+\tau_{a}^{b}\right) .
$$

The $\cong$ in $(20)$ is due to the influence of beam geometry and the effect of expansion/contraction on the properties 


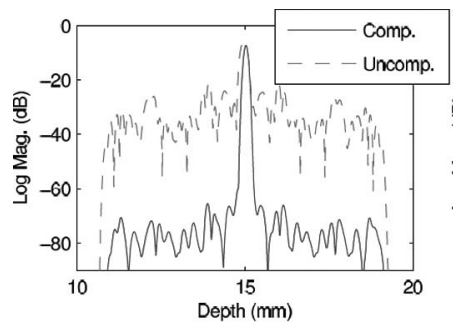

(a) Point scatterer at $15 \mathrm{~mm}$

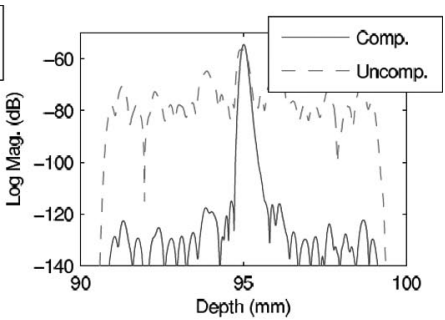

(b) Point scatterer at $95 \mathrm{~mm}$
Fig. 8. Effects of motion and compensation on simulated filter response envelopes at depths of $15 \mathrm{~mm}$ and $95 \mathrm{~mm}$.

of the medium. This may usually be disregarded for the minor perturbations caused by tissue motion between acquisition cycles.

Define reference received signals, complementary filter outputs, and complementary sums, respectively, as $S_{\mathrm{S}}(i, n, t), G_{\mathrm{S}}(i, n, t)$, and $C_{\mathrm{S}}(i, t), 1 \leq n \leq N$.

$$
\begin{gathered}
S_{\mathrm{S}}(i, n, t)=c(i+n, t) * B(t) * R(i, t)+N_{\mathrm{R}}(i, t) \\
G_{\mathrm{S}}(i, n, t)=F(i+n, t) * S_{\mathrm{S}}(i, n, t) \\
C_{\mathrm{S}}(i, t)=\sum_{n=1}^{N} G_{\mathrm{S}}(i, n, t) \\
=N \delta\left(t-N T_{\mathrm{b}}\right) * B(t) * R(i, t)+N_{\mathrm{C}}(i, t)
\end{gathered}
$$

Note that (4) and (5) have been used to simplify the indices somewhat. Combining (11) with (20), (21), and (22) allows us to write the sum of uncompensated filter outputs as

$$
\begin{aligned}
C_{\mathrm{U}}(i, t) & =\sum_{n=1}^{N} G_{\mathrm{S}}\left(i, n, t+\tau_{i}^{i-N+n}\right) \\
& =B(t)^{*} \sum_{a=i-N+1}^{i} c_{a a}(a, t)^{*} R\left(i, t+\tau_{i}^{a}\right)+N_{\mathrm{C}}(i, t) .
\end{aligned}
$$

The degree of misalignment, represented in (24) by the $\tau_{i}^{a}$ term, is a function of scatterer velocity and the time between acquisitions. This depends on the pulse repetition frequency $(\mathrm{PRF})$, which is limited by the maximum depth of interest and the length of the transmitted pulse. The time between acquisitions of lines along the same focal direction is a function of the PRF, the number of focal regions used on transmit, and the number of lines used to form the B-mode image.

The effects of motion were simulated in Matlab by updating the positions of individual scatterers between line acquisitions. The applied motion was sinusoidal in nature to approximate human respiration. A peak scatterer velocity of $6.2 \mathrm{~mm} / \mathrm{second}$ and angular velocity of $\pi / 3$ radians/ second were used based on figures published by Schlaijker et al. [23]. An initial phase of $\pi / 2$ radians was chosen to ensure the velocities simulated during the scan were close to the peak value.
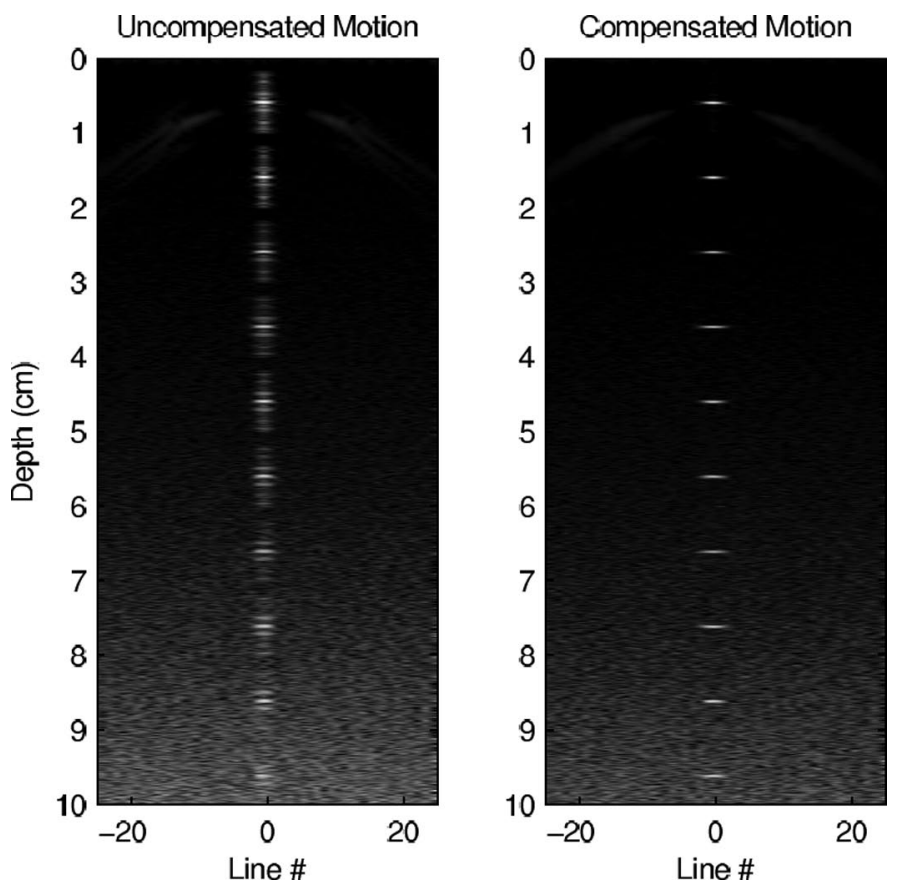

Fig. 9. Effects of motion and compensation on B-mode imaging using a Golay-encoded signal pair at $50 \mathrm{~dB}$ SNR.

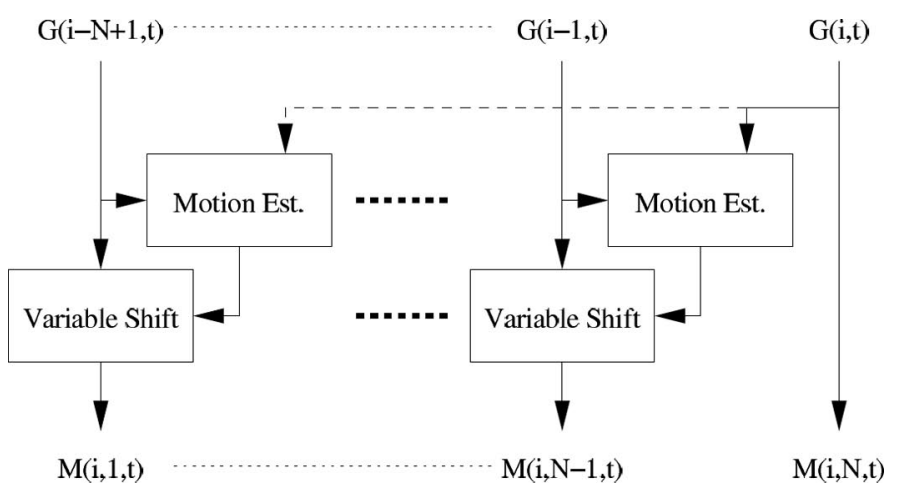

Fig. 10. Motion compensation block diagram.

The introduction of simulated axial motion caused sidelobe cancellation to break down, reducing compression and axial resolution and introducing the severe misalignment side-lobes evident in Fig. 8, in which the uncompensated envelopes exhibit side-lobes at -14 and $-10 \mathrm{~dB}$. These are manifested as artifacts in the corresponding B-mode image, which may be seen in Fig. 9.

A wide range of methods for ultrasonic velocity/motion estimation have been reported in the literature. In the current system, an interpolated estimate of the normalized cross-correlation maximum [24] is used to re-align the complementary filter outputs before summation, as illustrated in Fig. 10, although another wideband tissuemotion estimation algorithm could be used in its place [25], [26]. The reflections being cross-correlated are from different codes of the complementary set and their different side-lobe structures lead to a more defined (if slightly diminished) correlation peak. Log-linear gain correction was applied to the filtered data before motion estimation 


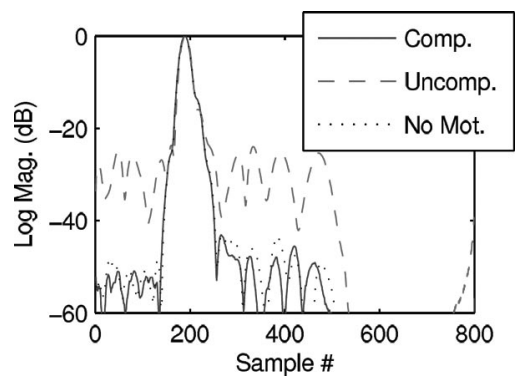

Fig. 11. Effects of motion and compensation on filter response envelopes obtained during experiments.

to prevent strong reflections from scattering centers close to the transducer from dominating the estimate.

If $\tilde{\tau}_{a}^{b}$ represents the estimated change in round-trip time due to motion between the ath and $b$ th acquisitions and $\varepsilon_{a}^{b}$ the estimate error, $\varepsilon_{a}^{b}=\tau_{a}^{b}-\tilde{\tau}_{a}^{b}$, the individual motioncompensated outputs are

$$
\begin{aligned}
M(i, n, t) & =G\left(i-N+n, t-\tilde{\tau}_{i}^{i-N+n}\right) \\
& =G_{\mathrm{S}}\left(i, n, t+\varepsilon_{i}^{i-N+n}\right) .
\end{aligned}
$$

The sum of compensated outputs is then

$$
\begin{aligned}
C(i, t) & =\sum_{n=1}^{N} M(i, n, t) \\
& =B(t) * \sum_{a=i-N+1}^{i} c_{a a}(a, t) * R\left(i, t+\varepsilon_{i}^{a}\right)+N_{\mathrm{C}}(i, t),
\end{aligned}
$$

which approaches (23) as the error term $\varepsilon$ approaches zero.

Complete compensation is unlikely in a typical imaging scenario but partial compensation is effective in reducing side-lobes to levels that compare favorably with other coding schemes proposed in the literature. In Fig. 8(b), compensation reduces peak misalignment side-lobes to $-62 \mathrm{~dB}$. The mean misalignment side-lobe level after compensation, averaged over the 10 point scatterers, is -67 dB. Fig. 9 illustrates the implications of this result for B-mode imaging. Compensation brings the motion artifacts below the visual threshold and the resulting images again demonstrate an increase in axial-resolution and noise-rejection compared with those for FM-chirp excitation. A comparison of the filter envelopes of Figs. 8(a) and 8 (b) suggests that the efficacy of compensation is unaffected by attenuation.

During experiments, data was captured using a single transducer and reflecting the signal from the wall of the tank. Motion was simulated by moving the transducer between transmissions ( $20 \mu \mathrm{m}$ in this instance), introducing motion side-lobes at about $-23 \mathrm{~dB}$. The solid line in Fig. 11 represents compensated data obtained experimentally. Here the compensated filter envelope exhibits lower sidelobes than the reference envelope obtained by transmitting both coded signals from the same position. The side-lobes
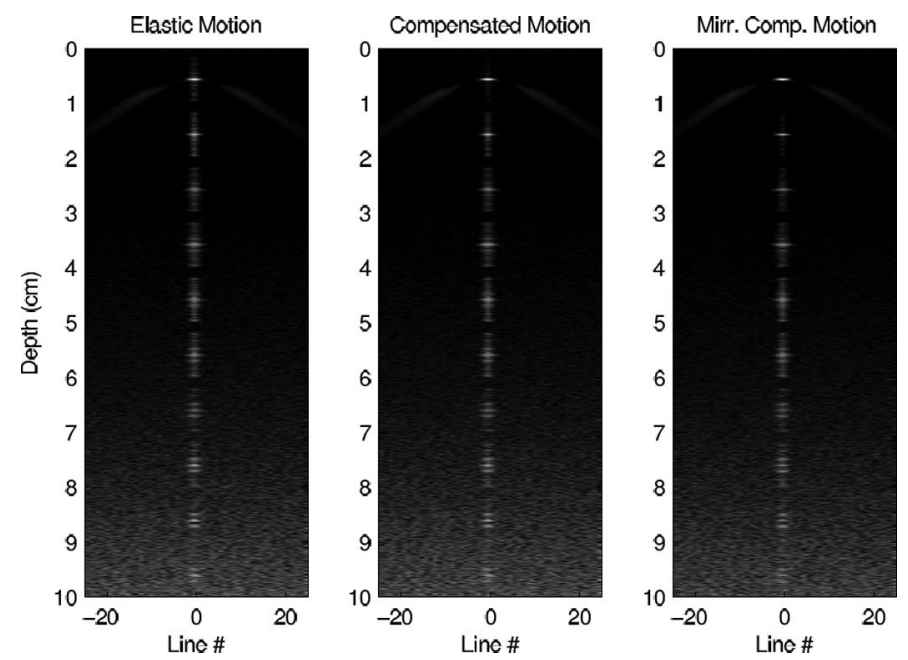

Fig. 12. Effects of inhomogeneous motion, single-segment compensation, and mirrored single-segment compensation on Golay-coded imaging. $50 \mathrm{~dB}$ SNR.

in the reference envelope are due to random timing jitter between multiple clock sources present in the experimental setup and would not be an issue in a clinical scanner using a single clock signal.

\section{Inhomogeneous Motion Compensation}

When performing a scan of a living subject, different regions of the beam may contain scatterers moving at different rates. This was simulated in Matlab by scaling the motion applied to individual scatterers in proportion to their axial distance from the transducer. In this situation, $R(i, t) \approx R\left(i-1, t+\tau_{i-1}^{i}\left(t / t_{\max }\right)\right)$.

The B-mode images in Fig. 12 were generated using simulated elastic motion and Golay coded excitation signals. Without compensation, misalignment side-lobe artifacts increase steadily with increasing distance from the transducer. Application of the motion compensation algorithm described in Section IX decreases the occurrence of side-lobe artifacts overall but significant motion artifacts remain.

\section{A. Multi-Segment Motion Compensation}

The two images at the left of Fig. 13 were generated by dividing each line of data into multiple segments (the examples given here used 5 and 10 segments, respectively) and compensating each segment individually. The simple line segmentation scheme employed here reduces the appearance of motion artifacts considerably.

\section{B. Mirrored Motion Compensation}

To reduce residual motion artifacts further, the mirrored compensation structure illustrated in Fig. 14 has been developed. Here, each output line is formed from the sum of two individually compensated sets of data. As 

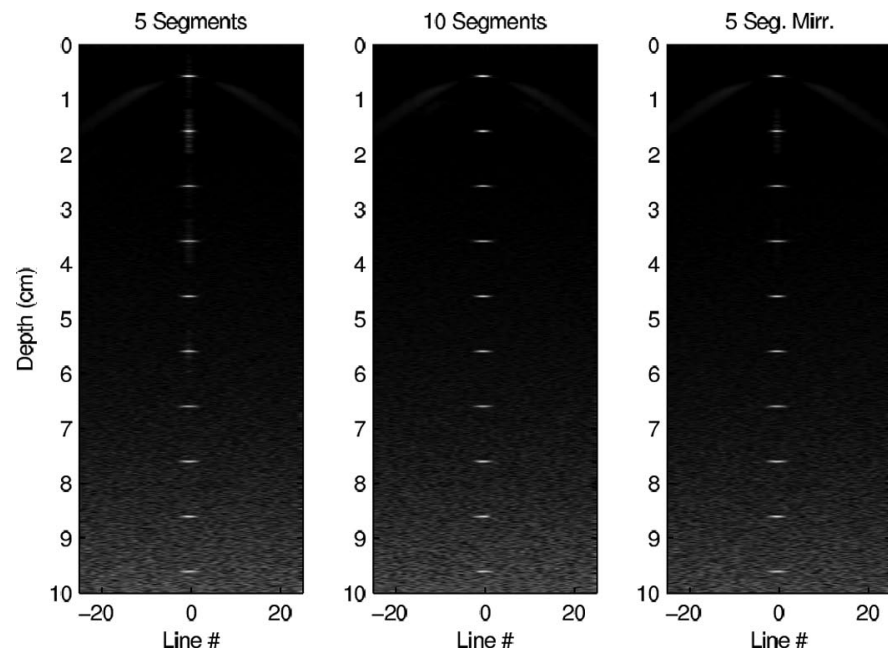

Fig. 13. Five- and ten-segment and mirrored 5-segment motion compensation applied to image data of Fig. 12.

\section{Stored Code Filter Outputs $G(n, t)$}

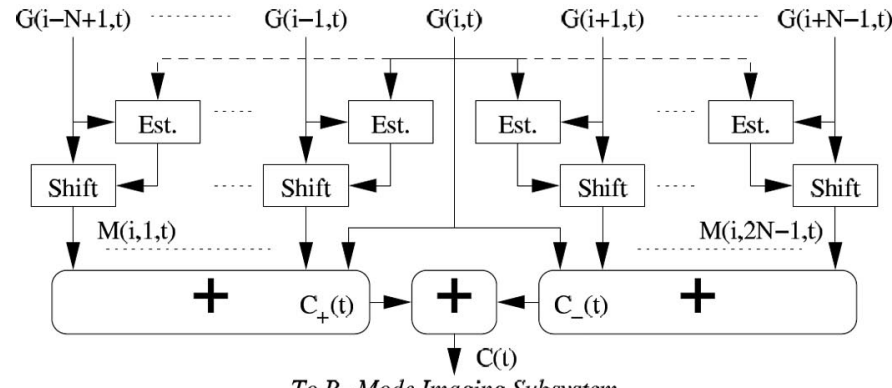

To B-Mode Imaging Subsystem

Fig. 14. Mirrored motion compensation for a single image line. Data from $2 N-1$ time-adjacent acquisitions for the line are used to form each compensated output. Data from the central (in time) acquisition are used as the reference for motion compensation.

each compensated line is based on a different set of motion estimates, any residual misalignment side-lobes tend not to coincide and indeed in some cases prove to interfere with one another destructively as in Fig. 15. Using this method, $2 N-1$ lines of received data are used to form each output. The two distinct compensated image lines are formed using the 1 st to $N$ th and $N$ th to $(2 N-1)$ th lines of complementary-filtered data. The central $(N$ th $)$ line of received data, common to both compensated lines, is used as the reference signal for both sets of motion estimates and the mirror-compensated output is formed by adding the individual compensated outputs.

Using this mirrored structure, the individual motion compensated outputs remain as defined in (25).

The mirrored sum of compensated outputs becomes

$$
\begin{aligned}
C_{\mathrm{M}}(i, t)= & \sum_{a=1}^{N} M(i, a, t)+\sum_{a=N}^{2 N-1} M(i, a, t) \\
\approx & 2 N \delta\left(t-N T_{\mathrm{b}}\right)^{*} B(t) * R(i, t) \\
& +\sum_{a=1}^{2 N-1} N_{\mathrm{G}}(i, t)+N_{\mathrm{G}}(N, t) .
\end{aligned}
$$

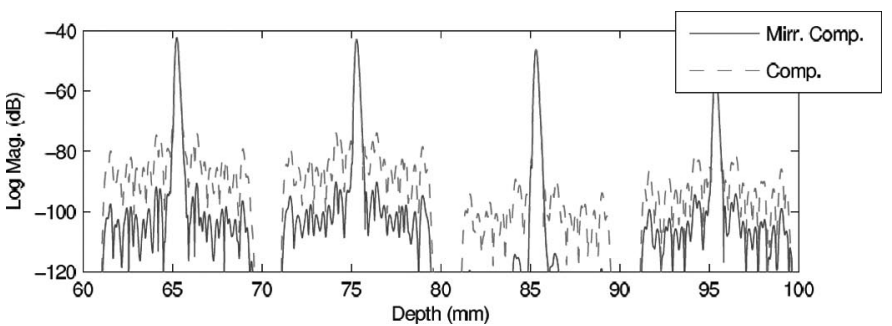

Fig. 15. Effects of mirrored compensation on 5-segment compensated filter envelopes. Residual misalignment side-lobes caused by partial motion compensation are reduced in magnitude.
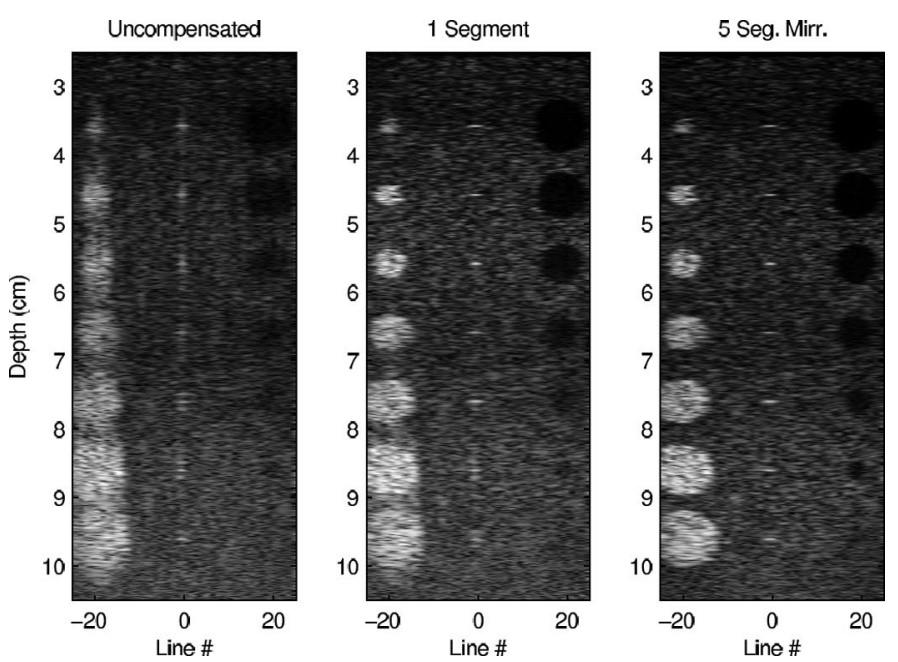

Fig. 16. Simulated images of a moving cyst phantom without motion compensation and with single-segment and mirrored five-segment compensation. Inhomogeneous motion and $45 \mathrm{~dB}$ SNR.

The coding gain for the mirrored output remains the same as for the individual compensated outputs because of the doubling of the $N_{\mathrm{G}}(N, t)$ term in (27). The filter peaks coincide because of the common compensation reference. The mirrored structure effectively spreads the effect of individual alignment errors over two frames, reducing their visual impact in any one frame. In some instances, misalignment side-lobes are opposite in phase and interfere destructively. The benefits are not consistently evident, but come at very little additional computational cost and can in some cases reduce artifacts considerably, as illustrated in Fig. 13. The residual misalignment side-lobes evident in the 5-segment compensated image on the left of the figure are no longer visible in the mirror-compensated image on the right. The three images in Fig. 16 were generated using Golay-coded excitation, the cyst phantom, and simulated elastic motion. The severe motion artifacts apparent in the uncompensated image are reduced but not eliminated by the application of single-segment motion compensation. Application of 5-segment mirrored motion compensation produces an image comparable to the reference image of a stationary phantom in Fig. 7 .

\section{CONCLUSIONS}

The proposed Golay-based scheme demonstrates promising performance under simulated conditions with an ap- 
preciable improvement in axial resolution compared with the linear FM-chirp, offers greater flexibility in choice of code-length than Barker-coded imaging, and eliminates the loss in frame-rate often associated with complementary-coded imaging. The side-lobe levels achievable using motion-compensated Golay-coded imaging are comparable to those of the LFM signals, and the increased resilience of the binary-filtered Golay codes to attenuation translates to a significant increase in coding gain at higher imaging depths. In addition, the technique is applicable to Golay complementary codes of any length, whereas the timebandwidth products of 50 or more required in order to achieve acceptable side-lobe levels using FM-coded signals could prove problematic in some imaging scenarios. Motion-compensation performance with simulated time-varying homogeneous motion of several point targets and of a simulated cyst phantom has been demonstrated to be very satisfactory. Inhomogeneous motion reduces the efficacy of compensation, but the segmentation and mirroring discussed in Section VI reduce visual artifacts significantly. The method appears to be particularly attractive for high imaging frequencies because of its robustness to the effects of frequency-dependent attenuation.

The preliminary experimental results presented in Section $\mathrm{V}$ and in greater detail in [27] suggest the methods could be successfully implemented in a commercial imaging system. Combining the method with the orthogonal code pairs and simultaneous transmission described in [9], [13], and [14] could potentially provide the benefits of complementary signal coding at twice the frame-rate of conventional B-mode imaging. In a system using multiple transmit foci, it may be appropriate to use several different code lengths. Shorter codes or uncoded pulses could be used for focal regions near the transducer to minimize the occurrence of residual misalignment artifacts and longer codes used for deep imaging to maximize coding gain. Future plans include simulation studies of an imaging system using multiple transmit foci and a code length that increases with depth, an assessment of the impact of various focusing and motion estimation parameters on the compensation process, and adaptation of the method for synthetic aperture imaging.

\section{ACKNOWLEDGMENTS}

The experimental work could not have been carried out without the assistance of M. Bennett and B. Lamboul at the Division of Medical and Radiological Sciences, University of Edinburgh.

\section{REFERENCES}

[1] R. Waag, J. Myklebust, W. Rhoads, and R. Gramiak, "Instrumentation for noninvasive cardiac chamber flow rate measurement," in Proc. IEEE Ultrasonics Symp., 1972, pp. 74-77.

[2] J. Hossack, "Introduction to the special issue on coded waveforms," IEEE Trans. Ultrason. Ferroelectr. Freq. Control, vol. 52, no. 2, pp. 158-159, Feb. 2005.
[3] M. O'Donnell, "Coded excitation system for improving the penetration of real-time phased-array imaging systems," IEEE Trans. Ultrason. Ferroelectr. Freq. Control, vol. 39, no. 3, pp. 341-351, May 1992.

[4] T. Misaridis and J. Jensen, "Use of modulated excitation signals in medical ultrasound. Part I: Basic concepts and expected benefits," IEEE Trans. Ultrason. Ferroelectr. Freq. Control, vol. 52, no. 2, pp. 177-191, Feb. 2005

[5] T. Misaridis and J. Jensen, "Use of modulated excitation signals in medical ultrasound. Part II: Design and performance for medical imaging applications," IEEE Trans. Ultrason. Ferroelectr. Freq. Control, vol. 52, no. 2, pp. 192-207, Feb. 2005.

[6] T. Misaridis and J. Jensen, "Use of modulated excitation signals in medical ultrasound. Part III: High frame rate imaging," IEEE Trans. Ultrason. Ferroelectr. Freq. Control, vol. 52, no. 2, pp. 208 219, Feb. 2005.

[7] F. Gran and J. Jensen, "Designing waveforms for temporal encoding using a frequency sampling method," Ultrasonics, Ferroelectrics and Frequency Control, IEEE Transactions on, vol. 54, no. 10, pp. 2070-2081, October. 2007.

[8] R. Bjerngaard and J. A. Jensen, "Should compression of coded waveforms be done before or after focusing? Proc. SPIE, vol. 4687, no. 1, pp. 47-58, 2002.

[9] R. Chiao and X. Hao, "Coded excitation for diagnostic ultrasound: A system developer's perspective," IEEE Trans. Ultrason. Ferroelectr. Freq. Control, vol. 52, no. 2, pp. 160-170, Feb. 2005.

[10] Y. Wang, K. Metzger, D. Stephens, G. Williams, S. Brownlie, and M. O'Donnell, "Coded excitation with spectrum inversion (CEXSI) for ultrasound array imaging," IEEE Trans. Ultrason. Ferroelectr. Freq. Control, vol. 50, no. 7, pp. 805-823, Jul. 2003.

[11] H. Zhao, L. Mo, and S. Gao, "Barker-coded ultrasound color flow imaging: Theoretical and practical design considerations," IEEE Trans. Ultrason. Ferroelectr. Freq. Control, vol. 54, no. 2, pp. 319 331, Feb. 2007.

[12] M. Golay, "Complementary series," IEEE Trans. Inf. Theory, vol. 7, no. 2, pp. 82-87, Apr. 1961.

[13] R. Chiao and L. Thomas, "Synthetic transmit aperture imaging using orthogonal Golay coded excitation," in Proc. IEEE Ultrasonics Symp., 2000, pp. 1677-1680.

[14] M.-H. Bae, W.-Y. Lee, M.-K. Jeong, and S.-J. Kwon, "Orthogonal Golay code based ultrasonic imaging without reducing frame rate," in Proc. IEEE Ultrasonics Symp., 2002, pp. 1705-1708.

[15] J. Gao and D. Liu, "Ultrasound flow motion detection using reverse Golay codes for decoding," in Proc. Int. Conf. Bioinformatics and Biomedical Engineering 2007, pp. 1253-1256.

[16] C. Leavens, R. Willams, P. Burns, and M. Sherar, "The use of phase codes in ultrasound imaging: SNR gain and bandwidth requirements," Appl. Acoust., vol. 70, no. 10, pp. 1340-1351, 2009.

[17] S. Nikolov, K. Gammelmark, and J. Jensen, "Recursive ultrasound imaging," in Proc. IEEE Ultrasonics Symp., vol. 2, 1999, pp. 16211625 .

[18] B.-H. Kim, G.-D. Kim, and T.-K. Song, "A post-compression based ultrasound imaging technique for simultaneous transmit multi-zone focusing," Ultrasonics, vol. 46, no. 2, pp. 148-154, 2007.

[19] J. Jensen, "Linear description of ultrasound imaging systems," Technical University of Denmark, Lyngby, Denmark, Tech. Rep., Jun. 2001

[20] P. Pallav, T. Gan, and D. Hutchins, "Elliptical-Tukey chirp signal for high-resolution, air-coupled ultrasonic imaging," IEEE Trans. Ultrason. Ferroelectr. Freq. Control, vol. 54, no. 8, pp. 1530-1540 Sep. 2007.

[21] C. Dolph, "A current distribution for broadside arrays which optimizes the relationship between beam width and side-lobe level," Proc. IRE, vol. 34, no. 6, pp. 335-348, Jun. 1946.

[22] P. Lynch, "The Dolph-Chebyshev window: A simple optimal filter," Proc. Am. Meteorol. Soc., vol. 125, no. 6, pp. 655-660, April. 1997.

[23] M. Schlaikjer, S. Torp-Pedersen, and J. Jensen, "Simulation of RF data with tissue motion for optimizing stationary echo canceling filters," Ultrasonics, vol. 41, pp. 415-419, 2003.

[24] S. Foster, P. Embree, and W. O'Brien Jr., "Flow velocity profile via time-domain correlation: error analysis and computer simulation," IEEE Trans. Ultrason. Ferroelectr. Freq. Control, vol. 37, no. 3, pp. 164-175, May. 1990.

25] S. Langeland, J. D'hooge, H. Torp, B. Bunens, and P. Suetens, "Comparison of time-domain displacement estimators for two-dimensional RF tracking," Ultrasound Med. Biol., vol. 29, no. 8, pp. 1177-1186, Apr. 2003. 
[26] F. Viola and W. Walker, "A spline-based algorithm for continuous timedelay estimation using sampled data," IEEE Trans. Ultrason. Ferroelectr. Freq. Control, vol. 52, no. 1, pp. 80-93, Jan. 2005.

[27] C. Cannon, "Motion compensation for complementary-coded ultrasonic imaging," Ph.D. dissertation, Institute for Digital Communication, School of Engineering, University of Edinburgh, UK, 2009.

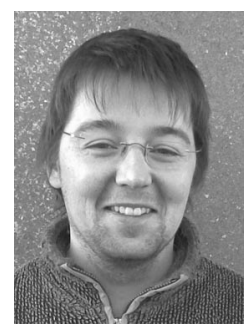

Cormac Cannon was born in London in 1978. He received the B.E. degree in electronic engineering from the National University of Ireland, Galway, in 2000 and an M.A. in traditional music performance from the University of Limerick in 2004.

Following graduation in 2000, he worked as a DSP hardware design and algorithm development engineer before taking up a research fellowship at MediaLabEurope in 2001, where he worked on haptic feedback for broadcast entertainment and new electronic musical interfaces. In the academic year 2004-2005 he taught music at the University of Limerick and studied reed-making for the uilleann pipes.

He enrolled as a Ph.D. candidate at the University of Edinburgh in 2005, investigating the use of coded excitation signals for medical ultrasonic imaging, and completed his Ph.D. studies in 2009.

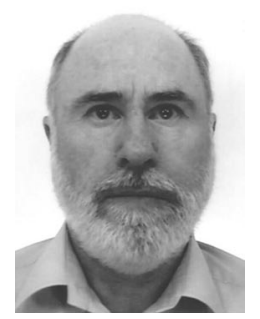

John Hannah received the B.Sc. degree in electrical engineering from the University of Strathclyde, Glasgow, UK, in 1970, and the Ph.D. degree from the University of Edinburgh, UK, in 1975. He was appointed a Lecturer in the Department of Electrical Engineering, University of Edinburgh, in 1975 . He is now a Senior Lecturer and a member of the Signals and Systems Research Group in the Department of Electronics and Electrical Engineering. He has undertaken research in a number of areas of signal processing. His current research interests include image processing and low-bandwidth video coding.

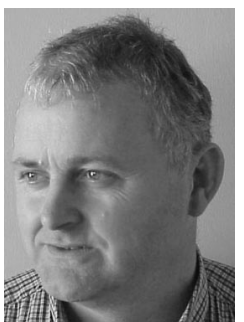

Steve McLaughlin (SM'04) was born in Clydebank, Scotland, in 1960. He received the B.Sc. degree in electronics and electrical engineering from the University of Glasgow, Glasgow, Scotland, in 1981 and the Ph.D. degree from the University of Edinburgh, Edinburgh, U.K., in 1989.

From 1981 to 1984, he was a Development Engineer with Barr \& Stroud Ltd., Glasgow, Scotland, where he was involved in the design and simulation of integrated thermal imaging and fire control systems. From 1984 to 1986, he worked on the design and development of high-frequency data communication systems with MEL Ltd., Dumfermline, Scotland, In 1986, he was a Research Fellow with the Department of Electronics and Electrical Engineering, University of Edinburgh, where he studied the performance of linear adaptive algorithms in high noise and non-stationary environments. In 1988, he joined the academic staff at Edinburgh, and from 1991 until 2001, he held a Royal Society University Research Fellowship to study nonlinear signal processing techniques. In 2002, he was awarded a personal Chair in Electronic Communication Systems at the University of Edinburgh. His research interests lie in the fields of adaptive signal processing and nonlinear dynamical systems theory and their applications to biomedical and communication systems.

Prof. McLaughlin is a Member of the Institution of Electrical Engineers and a Fellow of the Royal Society of Edinburgh. 